\title{
Laser scanning-based straightness measurement of precision bright steel rods at one point
}

\author{
Tobias Schmid-Schirling ${ }^{1}$ (D) $\cdot$ Lea Kraft $^{1} \cdot$ Daniel Carl $^{1}$
}

Received: 12 March 2021 / Accepted: 11 June 2021 / Published online: 9 July 2021

(C) The Author(s) 2021

\begin{abstract}
In industrial manufacturing of bright steel rods, one important quality factor is the straightness or straightness deviation. Depending on the application, deviations of less than $0.1 \mathrm{~mm}$ per meter rod length are desired and can be reached with state-of-the-art manufacturing equipment. Such high-quality requirements can only be guaranteed with continuous quality control. Manual straightness measurements conducted offline using a dial gauge provide accurate results on single positions of the rod. We propose a contactless, optical measurement technique based on laser scanning which has the potential to be used inline during production to inspect all rods over the entire length. Only for calibration of the system the rod needs to be turned around its axis. For the measurement of straightness deviation, it is not required to turn the rod. The method is based on evaluating the intensity signal of the reflected laser radiation against the scan angle. It is shown that in combination with an accurate calibration, this signal can be used to determine the rod's deviation from a straight rod. We explain the measurement and calibration principle as well as data evaluation. We present the experimental setup and first measurement results on a single position on several samples. For a homogeneous sample surface and neglecting laser drift, accuracy and precision were determined to be in the range of 10-20 $\mu \mathrm{m}$. We discuss the working principle of a potential inline system.
\end{abstract}

Keywords Straightness measurement $\cdot$ Steel rod $\cdot$ Contactless $\cdot$ Automated calibration

\section{Introduction to straightness measurement}

Bright steel rods are used for example in aerospace and automotive industries as axes and shafts. To manufacture bright steel rods with round cross section, crude steel is typically drawn or peeled. Straightness is one important quality feature of bright steel rods; respective tolerances are defined by DIN EN 10277 [1]. To specify straightness requirements for the production, a cylindrical tolerance zone with defined diameter may be provided [2].

One difficulty of the straightness measurement is the extreme ratio of length ( 2 to $12 \mathrm{~m}$ ) to diameter ( 5 to $70 \mathrm{~mm}$ ) in combination with high manufacturing accuracy with straightness deviations reaching less than $0.1 \mathrm{~mm}$ per meter rod length. Therefore, a straightness measurement system

Tobias Schmid-Schirling

tobias.schmid-schirling@ipm.fraunhofer.de

1 Fraunhofer Institute for Physical Measurement Techniques IPM, Georges-Köhler-Allee 301, 79110, Freiburg, Germany with corresponding measurement accuracy and precision is needed. Additionally, the bending of the rod due to gravity and the tendency to vibrate make the measurements technically more difficult.

The straightness deviation of bright steel rods may be determined manually [1] with a dial gauge on single rod positions and was extensively studied and modeled in [3]. Under laboratory conditions, a measurement accuracy of about $0.01 \mathrm{~mm}$ can be expected. Throughout the paper, we stick to the definition that straightness deviation is half the eccentricity when measured manually in the middle between two supports. Therefore, straightness deviation is the absolute value of deviation from a reference line defined by a perfectly straight rod.

In [4], the dial gauge was replaced by optical range finders oriented to the rod. Due to lack of time during production, it is not possible to realize a $100 \%$ inspection over the whole length of the steel rod. Another disadvantage is that the rod must be taken out of the production line for the measurement.

To address these shortcomings, an inline inspection system for straightness measurement is required. Several systems have been developed in the past. There exist 
commercial, optical measurement techniques based on laser line projection and application of the shadowing principle [5-8]. The achievable measurement error is sufficiently low under ideal conditions and for a single measurement position along the rod, no rotation of the rod is necessary. In order to evaluate the entire rod, the rod must either be moved inducing vibrations or the number of sensors must be increased.

Another option is to measure a two- or three-dimensional profile of a rod, e.g., by optical triangulation systems using a laser or other light types [9]. The rod is placed on a surface plate, rotating itself into static equilibrium due to its straightness deviation. From the measurement data, the straightness deviation is calculated. It is obvious that the principle is hard to apply to rather straight bars as the static equilibrium can not be reached.

Another proposed system uses stereoscopic image acquisition with three cameras combined with force sensors to measure the tracking force [10]. Under ideal conditions, the measurement error is likely to be sufficiently low. The drawback is that calibration process of the force sensors as well as data evaluation is complex.

In [11] and [12], the authors used the term spatial straightness deviation to highlight that also the direction of deviation should be taken into account. The proposed method in [12] is very accurate but hard to apply for a fast inline application.

Another example of measuring the straightness of long products is based on arc detection [13-18]. A laser projects a line onto the rod's surface forming an elliptical arc, which is captured by a camera and evaluated. The straightness measurement error is reported to be $0.2 \mathrm{~mm} / 2 \mathrm{~m} \mathrm{[13]} \mathrm{and}$ the measurement precision is about $0.06 \mathrm{~mm}$ [14]. The main advantages of this method are the possibility to integrate it into the production line and the short measurement time. The drawback is that the camera's field of sight limits the system. On the one hand, a variety of sensor pairs need to be placed along the rod which is a cost-intensive. On the other, the field of view limits the detected arc length for increased sample diameter which leads to an increase of the measurement error. The usage of cameras with a larger field of view leads to a reduction of the measurement precision of the system.

The straightness of rolled long products may be determined by measuring the contact force of oriented supports [19] or the spacing between the workpiece and mathematical reference line [20]. Another possibility to measure the straightness of tubular products is based on capacitance measurement [21]. Pulse generators are connected to electrically conductive strips that are covered by a layer of dielectric material. The measured capacitance is proportional to the distance of separation between the tube and the strips. From the description, it is clear that these methods are not designed to reach the required measurement accuracy and precision.

In this paper, we present a patented method [22] for measuring the straightness deviation of steel rods based on the laser scanning principle. The method does not require to rotate or move the rod for a measurement and is therefore potentially fast. The straightness deviation, which is optically measured, follows the definition described in [1]. In the first part of this paper, the measurement principle in one plane, the calibration procedure, and the prototype setup are described (chapter Section 2). It is shown that the setup can be calibrated with any rod without special requirements of its straightness. First straightness measurements and evaluation are described in Section 3. The measurement results are presented in Section 4 and their meaning for an inline measurement system are discussed in Section 5. Summary and conclusion is found in Section 6. The focus of this paper lies in the demonstration of the measurement principle at a single position on the rod, the estimation of the measurement accuracy, and precision of straightness measurements. The extension to the entire length of the rod is straight forward and explained in Section 5.

\section{Laser scanning-based straightness measurement}

\subsection{Measurement principle}

Figure 1 schematically shows the measurement principle as a cross-section of the setup at an arbitrary rod position. Two laser scanners are placed above the rod, which is located statically on mounting supports. Each laser scanner scans

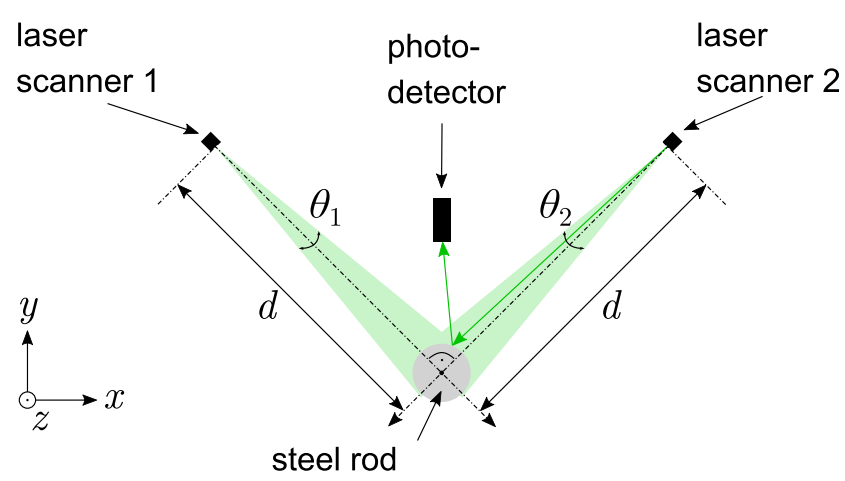

Fig. 1 Schematic cross-sectional view of the measurement setup. The scan angles are denoted by $\theta_{1,2}$ and the distance between the laser scanners and the rod is $d$. The laser scanners enclose an angle of about $90^{\circ}$. The rod is scanned by both lasers consecutively. The intensity of the light scattered off the rod's surface is recorded by a photodetector during scanning. The effect of a straightness deviation on the measurement signal is explained in Fig. 2 
(a)

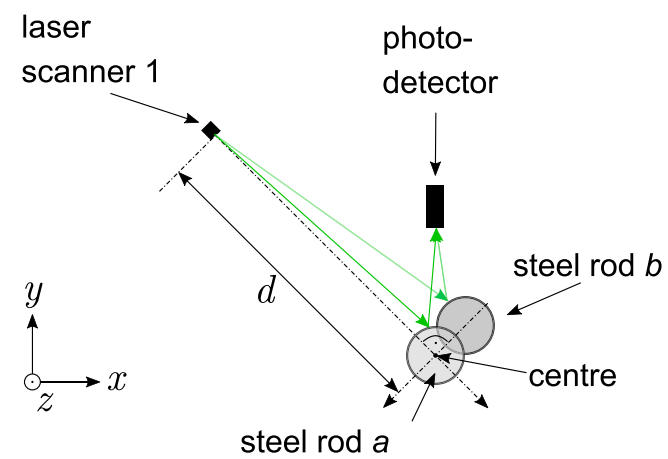

Fig. 2 Schematical illustration of the effect of straightness deviation on the measurement signal. a Cross-sectional view as in Fig. 1 but with two steel rods (straight and bend) and only one laser scanner for sake of clarity. A straightness deviation corresponds to a shift of the rod's

the laser beam perpendicular to the rod's axis across the rod (this corresponds to a small angular range of about $0,5^{\circ}$ ). The photodetector records the intensity of the laser light scattered off the rod's surface as a function of the scan angle. The two lasers scan the rod consecutively and thus the light intensity is recorded twice, for each laser scanner separately. In Fig. 2, it is schematically shown how a straightness deviation leads to a shift of the recorded intensity signal as a function of the scan angle for a single laser scanner: Let us assume steel $\operatorname{rod} a$ is straight and steel $\operatorname{rod} b$ has a straightness deviation. This results in a shift of its crosssection in the plane of projection (Fig. 2 left). The intensity signals of both rods show a bell-shaped curve with a clear peak (Fig. 2 right). For steel rod $b$, the intensity signal's position is shifted with respect to steel $\operatorname{rod} a$ as a function of the scan angle by an amount $\Delta \theta_{1}$ (for laser scanner 1).

From the shifts of the intensity signals $\Delta \theta_{1,2}$ (for laser scanner 1 and 2), the straightness deviation $s_{0}$ can be calculated as

$s_{\mathrm{o}}=\sqrt{s_{1}^{2}+s_{2}^{2}}$

with

$s_{1,2}=\tan \left(\Delta \theta_{1,2}\right) \cdot d$

This result is obtained without rotation of the rod. Also, from $s_{1}$ and $s_{2}$, not only the amplitude but also the direction of the straightness deviation can be deduced, which can be called spatial straightness deviation [12].

As the absolute position of the intensity signal is irrelevant, one can use any robust measure for its position. One possibility is to use the center of mass of the curve after smoothing the raw values to get rid of noise occuring in a real environment. For the same reason, bending due to gravity has no influence as long the setup itself is left unchanged (e.g. position of mounting supports). (b)

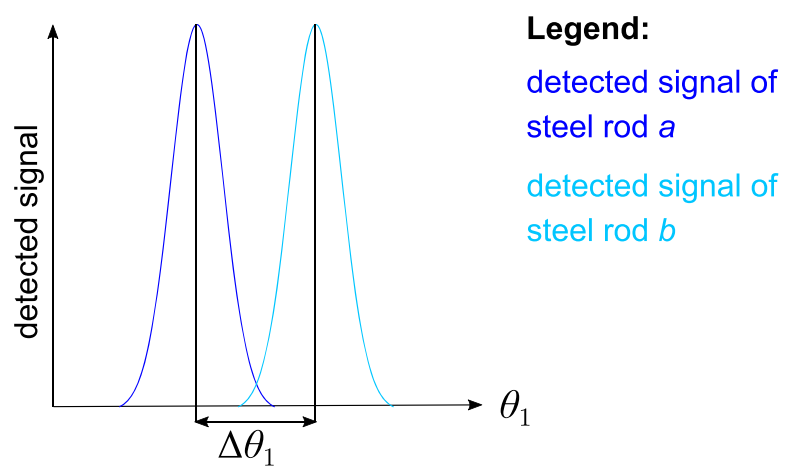

cross section in the plane of projection. b Recorded intensity signal for both rods. $\theta_{1}$ is the scan angle. $\Delta \theta_{1}$ is the shift between the peak positions of the detected signals due to the straightness deviation

The shape of the measured curve depends on various factors: rod diameter, laser spot size, distance to photodetector, aperture of the photodetector, and surface roughness of the rod. The signal's peak occurs where the scan angle $\theta_{1,2}$ of the laser scanner is such that the angle between the direction of specular reflection and the photodetectors aperture normal is minimal. For the determination of the intensity signal's position, both facts are irrelevant as long as the conditions in the setup are left unchanged.

As each laser scanner is only sensitive to a straightness deviation perpendicular to the scan axis, the two lasers scanning directions enclose $90^{\circ}$ for maximum sensitivity in all directions.

In order to scan the entire rod, the procedure described above has to be repeated at each position of the rod. This is easily achieved by a second beam deflection unit in each laser scanner, which redirects the laser beam along the rod in small steps. After each step, the procedure is executed and the straightness deviation of the rod at this position is obtained.

\subsection{Calibration principle}

The calculation of the straightness deviation according to (1) requires the knowledge of the intensity signal's position of a straight rod with the same diameter. This can be achieved by using a rod, whose straightness deviation is negligible for the desired application. In practice, this is hard to achieve. A simpler solution is to use any rod with unknown straightness deviation and the same diameter for calibration in combination with mounting supports, which turn the rod about its axis stepwise. After each step (e.g. $\left.10^{\circ}\right)$ a measurement is taken and the intensity signal's position for both laser scanners is calculated. After one complete rotation, the average of all calculated intensity signal's positions for each laser scanner (e.g. 36 positions 
per scanner) is a good approximation for the position that would have been deduced from an ideal straight rod in a single measurement. For an ideal straight rod, the 36 positions would all be the same. The position obtained is called calibrated position for the rest of the paper.

The straightness deviation of the rod, which was used for the calibration, can be calculated from the single calibration measurements itself. Every single measurement (obtained from both laser scanners but at the same rotation step) can be used to calculate the straightness deviation according to (1). The method is employed in this paper to assess measurement accuracy and precision of the experimental setup.

\subsection{Experimental setup}

Figure 3 shows the entire experimental setup. The steel rod lies on two mounting supports with variable distance $D$. The supports are coupled to a stepper motor to rotate the steel bar. A photomultiplier is placed vertically above the steel rod to detect the scattered light during the laser scan. The distance between the steel rod and the photomultiplier is approximately $1 \mathrm{~m}$. The distance $d$ from each laser scanner to the steel rod is approximately $2.6 \mathrm{~m}$. The laser scanners, photomultiplier, and electronic components like power supplies, ... are fixed to a common stiff structure. In a factory environment, one has to reinforce the structure in order to minimize vibrations.

The laser scanners and the photomultiplier are connected to a computer for automatic control and data acquisition. These components are controlled with analogue voltages; therefore, analogue/digital converters are placed closely to each of the components. A common clock signal is used for timing.

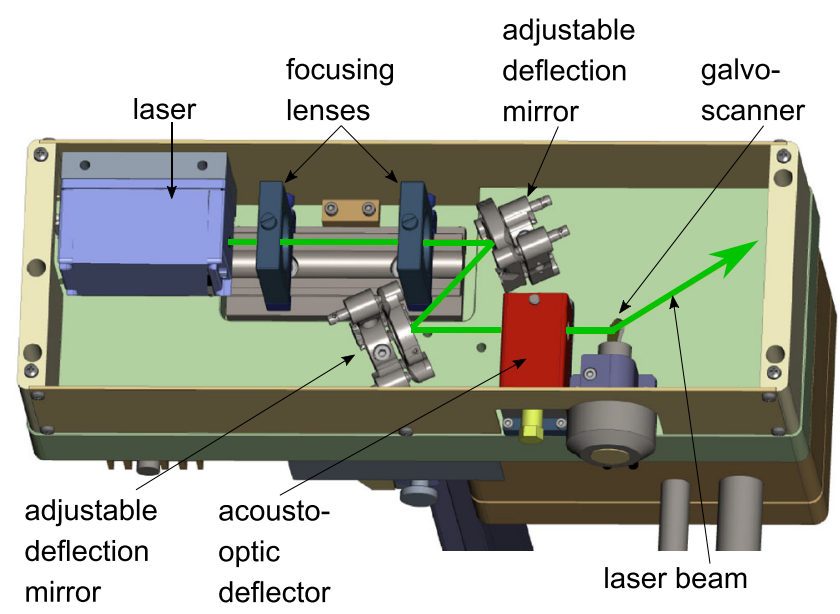

Fig. 4 Drawing of the beam path inside the laser scanner. The acoustooptic deflector (AOD) deflects the laser beam across and the galvo scanner deflects the laser beam along the steel rod. The focusing lenses are used to focus the laser spot accurately on the steel rod's surface. The adjustable deflection mirrors are used to position the laser beam in the middle of the AOD's entry aperture

The distance resolution on the steel rod surface due to the digital control resolution of the laser scanner and the distance $d$ is approximately $3.2 \mu \mathrm{m}$.

A dial gauge (Kaefer, model FKMD $12 \mathrm{~T}$ ) is used for reference straightness deviation measurements and is connected to the computer for automatic data acquisition. The stepper motor can be controlled from the same computer.

Figure 4 shows the interior of a laser scanner. As laser light source, a commercially available DPSS laser with wavelength $532 \mathrm{~nm}$ and beam quality factor $M^{2} \leq 1.1$ was chosen. The wavelength has a minor influence on
Fig. 3 Photo of the experimental setup. The steel rod lies on two mounting supports separated by a variable distance $D$. The supports are coupled to a rotation motor to turn the steel rod for calibration measurements. The laser scanners are placed above the steel rod with distance $d \approx 2.6 \mathrm{~m}$. The photomultiplier is mounted vertically above the steel rod and its aperture is directed onto the steel bar. A dial gauge for manual measurement is mounted to the setup for reference measurements. An exemplary, schematical laser light path is sketched as green arrows

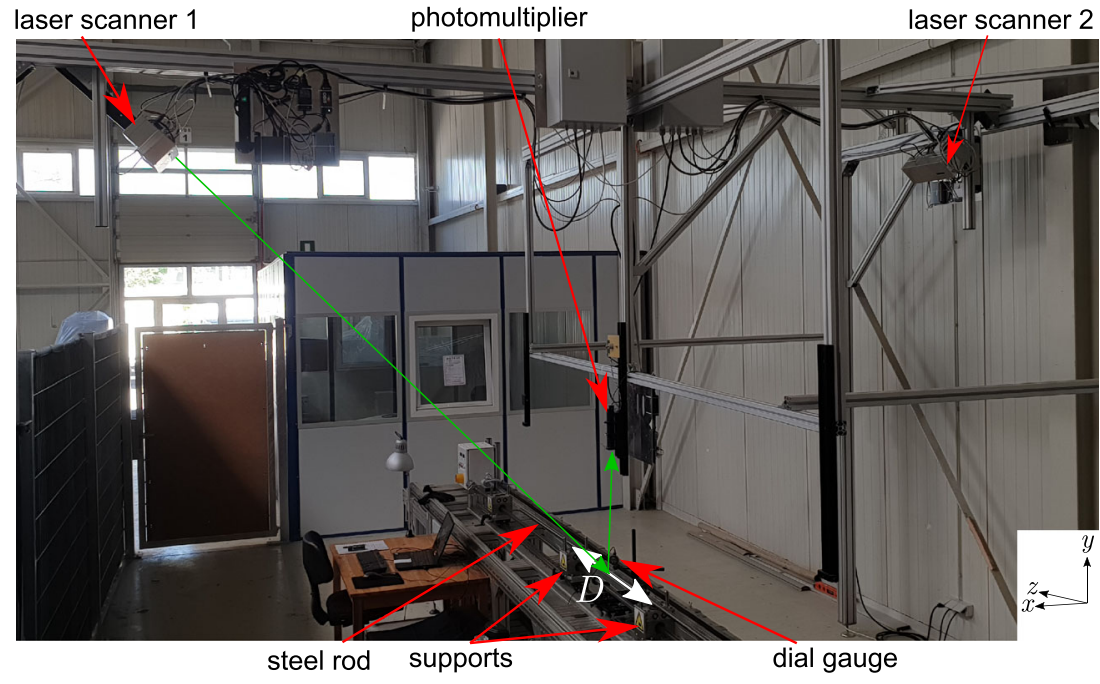


the measurement result but a low beam quality factor is important to get an sharp peak in the measurement signal (compare Fig. 2b). Two lenses are used to focus the laser beam on the rod's surface. Two adjustable mirrors are employed to position the laser beam onto the entry aperture of an acousto-optical deflector (AOD).

The AOD is used for scanning across the steel rod. It was chosen because a scan back and forth across the steel rod can be performed fast (the access time of the AOD is on the order of $1 \mu \mathrm{s}$ ) in comparison to a galvo scanner with mechanical inertia (small angle step response time approximately $100 \mu \mathrm{s}$ ). The device's small scanning angle of about $3^{\circ}$ is more than sufficient. The pointing precision of the device, which needs to be better than a few $\mu \mathrm{rad}$ for an accurate measurement of the straightness deviation, is limited only by the precision of the analogue control voltage, even without closed-loop control.

A galvo scanner is used to scan along the steel rod. For the measurements presented here, it was in a fixed position, such that the laser beam pointed roughly to the middle of the steel rod.

\section{Measurements}

\subsection{Steel rod samples}

The straightness deviation of three different steel rods was measured, see Fig. 5. The diameter of the first rod is $18 \mathrm{~mm}$. The visual appearance of its surface texture is shiny as it is chrome-plated in order to eliminate the influence of surface roughness. The second and third steel rod have a diameter of $20 \mathrm{~mm}$ and no surface finish. Due to handling of the samples, the surface shows additional surface defects like small dents, scratches, and scatter marks. The samples were chosen from industrial production without prior assessment of their straightness deviation.

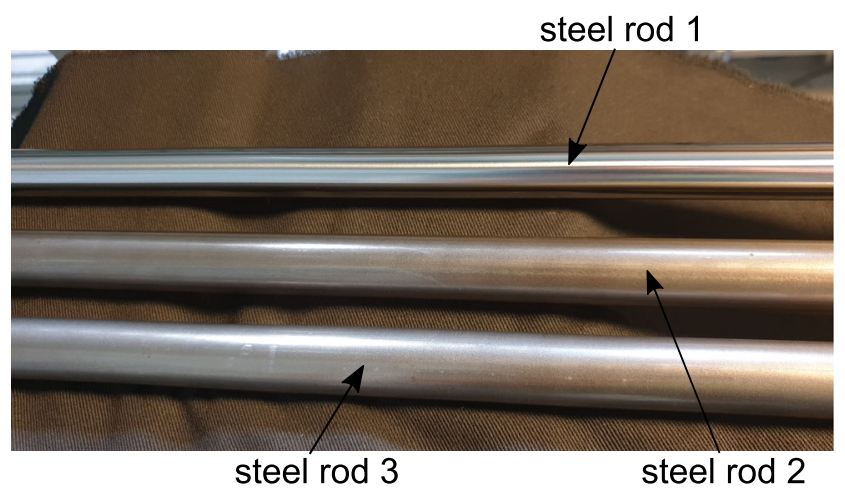

Fig. 5 Photo of the steel rod samples used for the measurements. The surface of steel rod 2 and 3 is rough and shows some surface defects in contrast to the surface of steel rod 1

\subsection{Experimental procedure and data evaluation}

Measurement data for each steel rod sample were acquired according to the following procedure: The two supporting mounts were fixed at the desired distance $D$ and the steel rod sample inserted. The sample was scanned 50 times at one position (roughly in the middle) by each laser scanner. Each scan consists of one forward and one backward scan movement, resulting in 100 measurement curves as shown in Fig. 6. Afterwards, the steel rod was turned by $10^{\circ}$ about its axis and the scanning procedure was repeated. In this way, 36 measurements were obtained. The measurement procedure was repeated four times leaving the samples on the supports.

For each scan, the measurement signal's peak position of the forward $\theta_{\text {forward }}$ and the backward $\theta_{\text {backward }}$ scan was determined, see Fig. 6. In order to eliminate the influence of noise, a polynomial regression was performed for the measurement values around the peak to obtain its position. The mean peak position $\theta_{\mathrm{p}}$ over the 50 scans is determined with (3) for each laser scanner separately.

$\theta_{\mathrm{p}}=\frac{1}{2 \cdot 50}\left(\sum_{i=1}^{50} \theta_{\text {forward }, i}+\sum_{i=1}^{50} \theta_{\text {backward }, i}\right)$

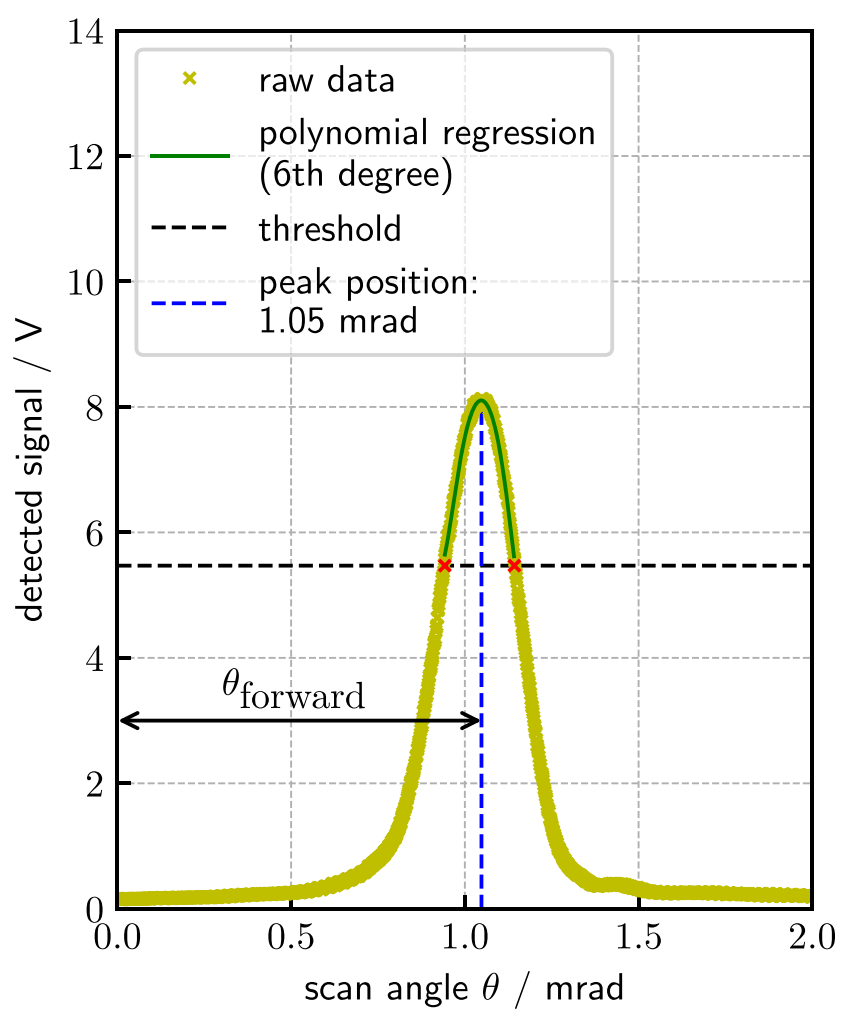

Fig. 6 Exemplary illustration of the determination of the peak position of a single forward scan. A 6th order polynomial regression on the upper third of the raw data was empirically found to match the raw data closely enough to eliminate the influence of noise 
The averages $\bar{\theta}_{\mathrm{p}}$ of the 36 values for each measurement series are taken as calibration position. Equation 1 is employed to calculate the straightness deviation $s_{\mathrm{O}}$ with

$$
\Delta \theta_{1,2}=\bar{\theta}_{\mathrm{p}}-\theta_{\mathrm{p}}
$$

for each rotation step. The total 144 straightness deviation values per sample and support distance position were averaged to obtain a mean straightness deviation $\bar{s}_{0}$.

Reference values for each sample and mounting support distance $D$ were obtained using the dial gauge according to [1]. These reference values were used to assess the accuracy of the laser scanning method. Four repeated measurements were taken leaving the samples on the supports but positioning the dial gauge again. The measurement position on the sample was approximately the same as in the optical measurement. $n=36$ single measurements are read out of the dial gauge during one rotation. Half of the difference between the minimum and maximum dial gauge value is the measured straightness deviation. Finally, the arithmetic mean $\bar{s}_{\mathrm{m}}$ and its standard error of the four measurements are used for comparison with laser scanning method's results.

(a)

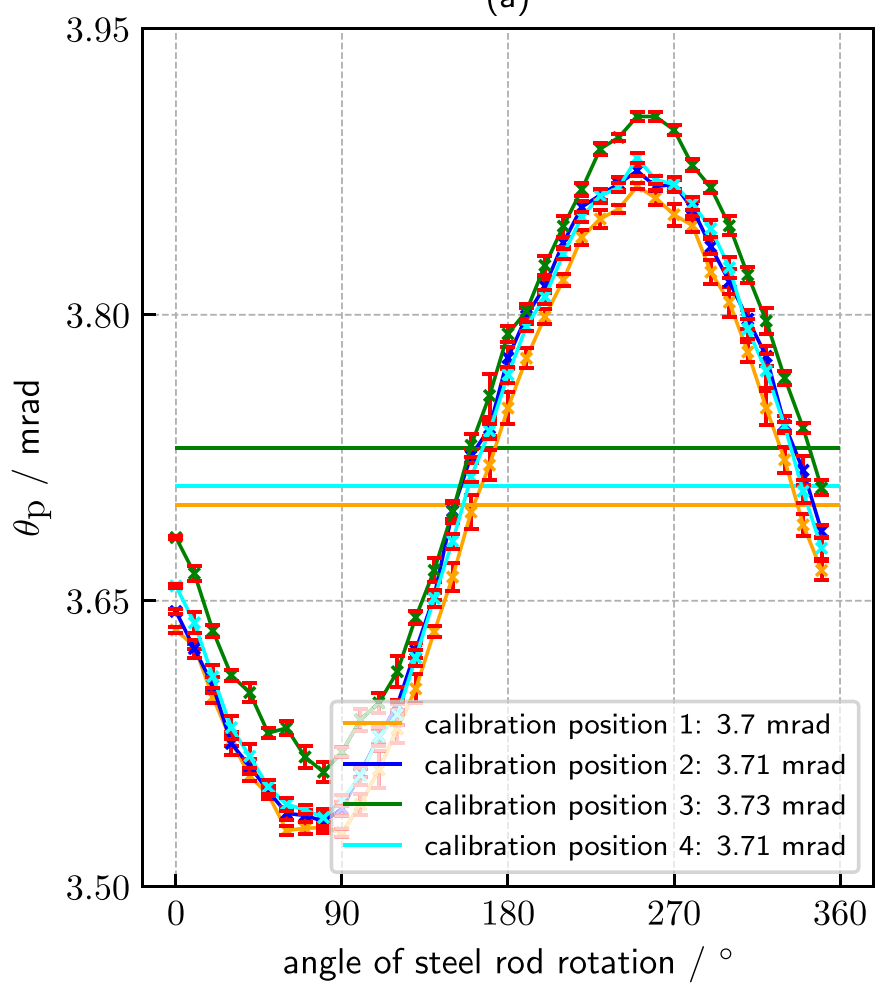

Fig. 7 Exemplary plots of mean peak positions $\theta_{\mathrm{p}}$ for a laser scanner 1 b laser scanner 2 versus angle of rotation of the sample. Four series with 36 data points each are shown for each laser scanner. The series are recorded consecutively leaving the sample on the supports. Error bars indicate the standard deviation of 100 single scans ( 50 forward

\section{Results}

In Fig. 7, the combined measurements for sample 1, both laser scanners, four complete rotations and $D=$ $1.58 \mathrm{~m}$ are shown. The sine shape of the curves indicate the displacement of the steel rod during rotation due to straightness deviation. The data points obtained from laser scanner 2 are shifted approximately by $90^{\circ}$ with respect to the curves obtained by laser scanner 1 . This is due to the fact that the laser scanners enclose an angle of about $90^{\circ}$. Similar curves were obtained for sample 2 and 3 .

The error bars plotted in Fig. 7 are the standard deviation of 100 single scans, giving an indication of statistical noise. Statistical noise may be present due to environmental influences like air fluctuations, dust particles in the air, vibrations, and residual noise in the electronics, e.g. the photomultiplier. The error bars are much smaller than deviations of single data points from an ideal smooth curve. This indicates that fewer scans are sufficient for precise results. Deviations from the ideal smooth curve are mainly due to surface inhomogeneity (scratches, stains, dust particles, ...).

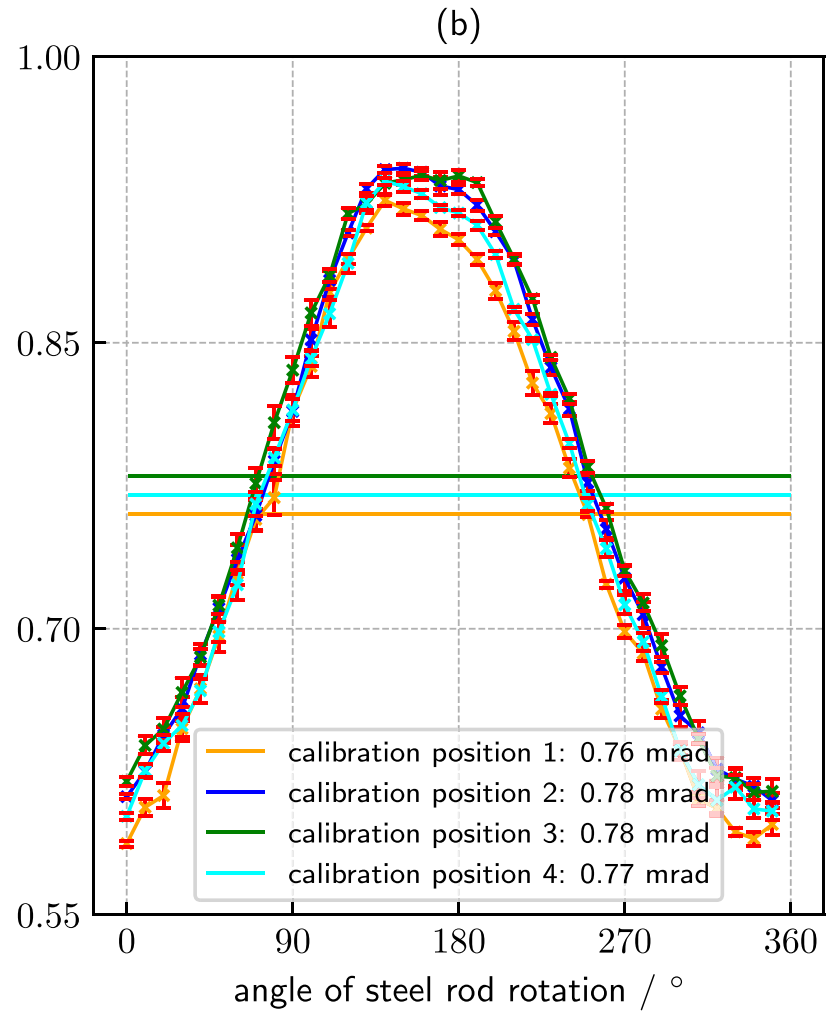

and 50 backward scans). The lines between data points are plotted for the sake of clarity. The mean value for each series $\bar{\theta}_{\mathrm{p}}$ is called calibration position, indicated with a horizontal line and numerically given in the boxes at the bottom 
Table 1 Straightness measurement results

\begin{tabular}{|c|c|c|c|c|c|c|c|c|c|c|}
\hline \multirow[b]{2}{*}{$D / \mathrm{m}$} & \multirow[b]{2}{*}{$d / \mathrm{m}$} & \multicolumn{2}{|c|}{ Steel rod 1} & \multirow{2}{*}{$\frac{\text { Steel rod } 2}{\sigma_{\mathrm{s}} / \mu \mathrm{m}}$} & \multicolumn{6}{|c|}{ Steel rod 3} \\
\hline & & $\bar{s}_{\mathrm{m}} / \mu \mathrm{m}$ & $\bar{s}_{\mathrm{o}} / \mu \mathrm{m}$ & & $\bar{s}_{\mathrm{m}} / \mu \mathrm{m}$ & $\bar{s}_{\mathrm{o}} / \mu \mathrm{m}$ & $\sigma_{\mathrm{s}} / \mu \mathrm{m}$ & $\bar{s}_{\mathrm{m}} / \mu \mathrm{m}$ & $\bar{s}_{\mathrm{o}} / \mu \mathrm{m}$ & $\sigma_{\mathrm{s}} / \mu \mathrm{m}$ \\
\hline 0.50 & 2.57 & $57 \pm 1$ & $56 \pm 2$ & 15 & $44 \pm 1$ & $70 \pm 3$ & 41 & $71 \pm 1$ & $92 \pm 7$ & 45 \\
\hline 1.00 & 2.57 & $242 \pm 1$ & $222 \pm 3$ & 22 & $163 \pm 1$ & $165 \pm 1$ & 39 & $292 \pm 1$ & $276 \pm 9$ & 42 \\
\hline 1.58 & 2.62 & $448 \pm 4$ & $437 \pm 2$ & 16 & $334 \pm 2$ & $321 \pm 7$ & 39 & $435 \pm 2$ & $435 \pm 9$ & 55 \\
\hline
\end{tabular}

$D$ is the distance between supports and $d$ the distance between each laser scanner and the steel rod. $\bar{s}_{\mathrm{m}}$ is the reference value acquired from the manual dial gauge measurement. $\bar{s}_{\mathrm{O}}$ is the mean value of four optical straightness measurements including one complete steel rod rotation each and its standard deviation from these four values. $\sigma_{\mathrm{s}}$ is the standard deviation of one optical measurement calculated from the 36 straightness measurement values each acquired at one fixed rotational position

The calibration positions have a variability of 30 $\mu \mathrm{rad}$ at maximum. The reason was found to be an unsufficient pointing stability of the laser $(<30 \mu \mathrm{rad}$ for $\pm 3^{\circ} \mathrm{C}$ according to specs). If using the laser scanner 1 measurements, e.g. from series 1 with the calibration position of series 3, the resulting measurement error of the straightness deviation is larger than $70 \mu \mathrm{m}$. As this is much larger than the measurement error we are aiming at, measures for improving the pointing stability have to be taken in the future. For the results presented here, this source of measurement error has a minor effect as each straightness deviation and the calibration position were calculated from the same measurements.

The straightness measurement results for the three steel rods can be found in Table 1. A single straightness deviation measurement is done without rotating the bar. The values provided here were obtained by averaging over different rotation positions to assess the accuracy of the method.

With increasing support distance $D$, the measured straightness deviation increases as the rod's bending leads to a larger measurable sag. In this way, we were able to test the method over a wider range of straightness deviations without the need of more samples. For the largest support distance, the laser scanners had to be readjusted slightly as the scan angles $\theta$ were not sufficient due to the large sag leading to a slightly different distance $d$.

$\bar{s}_{\mathrm{O}}$ is the mean straightness deviation provided with the standard deviation of the four measurement series. This standard deviation indicates the contribution of the calibration procedure to the entire uncertainty budget of the straightness measurement. It is $9 \mu \mathrm{m}$ maximum and $5 \mu \mathrm{m}$ on average. As the calibration was renewed before each of the four series, it is not dominated by the pointing instability of the lasers.

$\sigma_{\mathrm{s}}$ is the mean of four standard deviations, each calculated from the 36 single measurements of a measurement series. This standard deviation indicates the precision of a single straightness measurement. It is $55 \mu \mathrm{m}$ maximum and $35 \mu \mathrm{m}$ on average. It is noticeable that $\sigma_{\mathrm{s}}$ is significantly larger for steel rod 2 and 3 (44 $\mu \mathrm{m}$ on average) than for steel rod 1 (18 $\mu \mathrm{m}$ on average). Inspection of single measurement curves indicated that this is mainly due to the surface inhomogeneity, leading to distorted measurement curves. This limits the accuracy of the peak position's determination. The amount and extent of surface inhomogeneity existent on steel rod 2 and 3 is unlikely to occur directly after manufacturing.

For further optimization of the measurement system, one should inspect the resulting measurement curves closely to determine the most robust method for the determination of the intensity signals's position. Here, we used a 6th order polynomial regression to determine the peak position merely for sake of simplicity.

The accuracy of the method can be assessed by the deviation between manual and optical measurement. It is $26 \mu \mathrm{m}$ maximum and $12 \mu \mathrm{m}$ on average (not shown in Table 1).

From the manual measurements, it can be deduced that steel rod 2 shows the smallest straightness deviation, a result that is also achieved with the optical measurements except for the measurement with $D=0.50 \mathrm{~m}$. For smaller support distances $D$, the measurable sag becomes smaller. Thus, the differences in straightness deviation between the rods become smaller, too. For $D=0.50 \mathrm{~m}$, the differences in straightness deviation are in the order of the methods accuracy.

\section{Discussion}

The aim of the study was to assess if the proposed optical method is suitable for the inline measurement of straightness deviations of turned or drawn bright steel rods in accordance with [1]. Therefore, the method has to be accurate, precise, and fast. Straightness deviations should be measurable with a measurement error smaller than $0.1 \mathrm{~mm}$ and the measurement for the entire rod should not take more than few seconds. 
With the results obtained here, we believe that the method has the potential to fulfill such requirements in the future. The presented accuracy $(0.012 \mathrm{~mm}$ on average) and precision of a single measurement $(0.018 \mathrm{~mm}$ on average for a homogeneous sample surface) appear promising. The determination of a single measurement took about $0.8 \mathrm{~s}$. For an entire steel rod of several meters length, this is clearly to slow. But as discussed (see Fig. 7), much fewer scans on a single position should be sufficient without affecting the precision and therefore opening potential for speed up.

Figure 8 shows the idea of an inline measurement system. During the straightness measurement procedure, the steel rod is scanned by a rectangular shaped signal of the two laser scanners. Thus, AOD and galvo scanner shift the laser beam alternating. Several photodetectors are mounted above the sample. The scan angle of the galvo and the acceptance angle of the photodetectors should be as large as possible to minimize the required number of hardware components. All optical components have to be mounted on solid structures to minimize vibrations. Vibrations of the laser scanners as well as air turbulences decrease the precision of the measurement result and therefore require more measurements, which in turn cause the measurement time to increase. To achieve the mentioned accuracy, the pointing stability of the lasers has to be improved significantly. This may be achieved by active thermal control of the laser or a monitor diode measuring the drift.

The method not only gives the straightness deviation as absolute value but also the direction and therefore the spatial straightness deviation at one point (in contrast to the manual measurement with a single dial gauge). From the measurement of the entire rod length, one could calculate the spatial straightness deviation for the entire rod using, e.g., least square methods [12]. This helps to eliminate

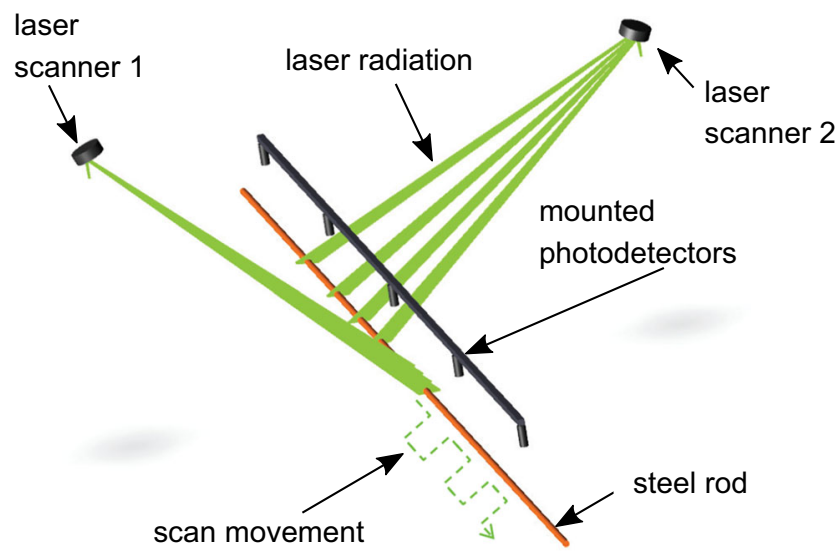

Fig. 8 Sketched inline straightness measurement setup with required components. The steel rod is scanned by a rectangular shaped signal of each laser scanner one by one. Photomultiplier are mounted above the steel rod and detect the reflected and scattered laser radiation outliers on single positions automatically. This may further help to increase the precision and reduce measurement time.

With such a system, not only round steel bars could be inspected. When scanned across, any profile leads to a characteristic curve whose position may be assessed with respect to a calibrated position for a straight profile. Depending on the profile, the calibration procedure explained in Section 2.2 may not be applicable.

\section{Conclusion and outlook}

In this study, the straightness deviation of three bright steel rods has been measured with a non-contact laser scanning approach. The approach is based on the analysis of the relative position of the laser beam intensity reflected off the steel rod's surface with respect to the scanning position. Additionally, a calibration procedure of such a device was proposed.

Accuracy and precision were found to be acceptable $(0.012 \mu \mathrm{m}$ and $0.018 \mu \mathrm{m}$ for a homogeneous sample surface, respectively). The measurement time at a single position was about $0.8 \mathrm{~s}$. The potential for improvement was discussed.

As next steps towards a prototype for an inline measurement system, the following tasks were identified. First, the pointing stability of the lasers has to be improved. Second, other methods for robustly determining the signal's position (correlation, other curves for regression, ...) have to be investigated and compared. Third, the method has to be tested over the entire length of a steel rod and the results compared.

Acknowledgements The authors would like to thank Joachim Anders, Volker Jetter, and Michael Seib for support in optomechanical engineering, and Ralf Singler for support in electronical engeneering of the experimental setup.

Author contribution Tobias Schmid-Schirling: conception, analysis, interpretation, writing - original draft preparation/review and editing. Lea Kraft: conception, execute trials, analysis, visualization—original draft preparation.

Daniel Carl: writing-review and editing, supervision.

Funding Open Access funding enabled and organized by Projekt DEAL. This work was supported by the Fraunhofer Internal Programmes under Grant No. MEF 600865.

\section{Declarations}

Conflict of Interest The authors declare no competing interests.

Open Access This article is licensed under a Creative Commons Attribution 4.0 International License, which permits use, sharing, adaptation, distribution and reproduction in any medium or format, as long as you give appropriate credit to the original author(s) and the source, provide a link to the Creative Commons licence, and indicate if changes were made. The images or other third party material in this 
article are included in the article's Creative Commons licence, unless indicated otherwise in a credit line to the material. If material is not included in the article's Creative Commons licence and your intended use is not permitted by statutory regulation or exceeds the permitted use, you will need to obtain permission directly from the copyright holder. To view a copy of this licence, visit http://creativecommons. org/licenses/by/4.0/.

\section{References}

1. Bright steel products (2018) Technical delivery conditions. DIN EN 10277

2. Geometrical Product Specifications (GPS) (2004) Geometrical tolerancing - tolerances of form, orientation, location and run-out. ISO 1101

3. Fangmeier R (1976) Geradheit beim Richten von blankem Rundstahl. Stahl und Eisen 96:872-878

4. Parker MA, Ahmed HJ (1983) Nuclear fuel rod straightness measuring system and method. US4589082A

5. Zumbach Electronic GmbH (2020) BENDCHECK - In-line straightness measurement using precise non-contact laser diameter gauges. https://www.zumbach.com/pdf/Literature/ $\backslash$ BENDCHECK/Bendcheck_ODAC.004.0009.EN.pdf. Accessed 05 Jan 2021

6. (2020) Straightness Check - Quality control for long products. https://www.lap-laser.com/fileadmin/user_upload/en/products/laser_ measurement/long_products/straightness_check/flyer_straightness_ check.pdf. Accessed 05 Jan 2021

7. GmbH Laser Applikationen LAP (2012) Stabstahl auf Geradheit getestet. Draht 63-55

8. Reiter M (2013) Verfahren und Vorrichtung zum prüfen von Rohren. DE 102011119658

9. Fumagalli L, Gregorelli E, Tomassini P (2016) Measuring method and device to measure the straightness error of bars and pipes. EP3093611

10. Kavousian A (2017) Device and method for measuring the straightness of a rod-like work piece. US 2017 / 0284798 A1

11. Zhu L (2010) Estimation of uncertainty in spatial straightness measurement according to next generation of GPS standard. Third
International Workshop on Advanced Computational Intelligence 666-669. https://doi.org/10.1109/IWACI.2010.5585140

12. He M, Ye X, Li X, Gan X (2013) Evaluation of spatial straightness error using laserTRACER. Proc SPIE 8759 87593S. https://doi.org/10.1117/12.2014642

13. Sun C, You Q, Ye S (2001) Online machine vision method for measuring the diameter and straightness of seamless steel pipes. Opt Eng 40(11). https://doi.org/10.1117/1.1412424

14. Lu RS, Li YF, Yu Q (2001) On-line measurement of the straightness of seamless steel pipes using machine vision technique. Sensors Actuat A Phys 94:95-101. https://doi.org/10.1016/S09244247(01)00683-5

15. Lee HW, Hsu PR, Pan SP, Liu TA, Liou HC (2015) Applying structured light on cylinder straightness detection using break line method applied mechanics and materials. 764-765:12981303. https://doi.org/10.4028/www.scientific.net/AMM.764-765. 1298

16. Wu L, Wen G, Wang Y, Xu X (2020) An enhanced detection system of drill rod bending degree based on two-dimensional laser. Sensors 20:370. https://doi.org/10.3390/s20020370

17. Wu B, Xue T, Zhang T, Ye S (2010) A novel method for round steel measurement with a multi-line structured light vision sensor. Meas Sci Technol 21:025204. https://doi.org/10.1088/0957-0233/ 21/2/025204

18. Schalk P, Ofner R, O’Leary P (2007) Pipe eccentricity measurement using laser triangulation. Image Vis Comput 25:1194-1203. https://doi.org/10.1016/j.imavis.2006.04.021

19. Schwenzfeier W, Gahleitner J, Wollendorfer G, Naumann N (2003) Verfahren und Vorrichtung zur Bestimmung der Krümmung von Langgut. AT410977B

20. Daube T, Nerzak T (2009) Measuring straightness of an elongated rolled workpiece. US2009/0044583A1

21. Luebke JT (1989) Method and apparatus for measuring the straightness of tubes. US4851760A

22. Carl D, Jetter V, Schmid-Schirling $T$ (2019) Device for contactlessly determining the straightness of at least one long product and method for calibrating a device of this kind. WO2019/001738AI

Publisher's note Springer Nature remains neutral with regard to jurisdictional claims in published maps and institutional affiliations. 